PROCEEDINGS OF THE

AMERICAN MATHEMATICAL SOCIETY

Volume 129, Number 3, Pages 739-747

S 0002-9939(00)05718-X

Article electronically published on September 19, 2000

\title{
COMPACT OPERATORS ON THE BERGMAN SPACE OF MULTIPLY-CONNECTED DOMAINS
}

\author{
ROBERTO RAIMONDO
}

(Communicated by Joseph A. Ball)

\begin{abstract}
If $\Omega$ is a smoothly bounded multiply-connected domain in the complex plane and $A=\sum_{j=1}^{m} \prod_{k=1}^{m_{j}} T_{\varphi_{j, k}}$, where $\varphi_{j, k} \in L^{\infty}(\Omega, d \nu)$, we show that $A$ is compact if and only if its Berezin transform vanishes at the boundary.
\end{abstract}

\section{INTRODUCTION}

Let $\Omega$ be a bounded multiply-connected domain in the complex plane $\mathbf{C}$, whose boundary $\partial \Omega$ consists of finitely many simple closed smooth analytic curves. For $d \nu=\frac{1}{\pi} d x d y$ we consider the usual $L^{2}$-space $L^{2}(\Omega)=L^{2}(\Omega, d \nu)$. The Bergman Space $H^{2}(\Omega, d \nu)$, consisting of all holomorphic functions which are $L^{2}$-integrable, is a closed subspace of $L^{2}(\Omega, d \nu)$. The Bergman Projection is the orthogonal projection $P: L^{2}(\Omega, d \nu) \longrightarrow H^{2}(\Omega, d \nu)$. It is well known that for any $f \in L^{2}(\Omega, d \nu)$ we have

$$
\operatorname{Pf}(w)=\int_{\Omega} f(z) \overline{K^{\Omega}(z, w)} d \nu(z),
$$

where $K^{\Omega}$ is the Bergman reproducing kernel of $\Omega$. For $\varphi \in L^{\infty}(\Omega, d \nu)$ the Toeplitz operator $T_{\varphi}$ from $H^{2}(\Omega, d \nu)$ to itself is defined by $T_{\varphi}=P M_{\varphi}$, where $M_{\varphi}$ is the standard multiplication operator.

Axler and Zheng have proved (see [2]) that if $D$ is the disk $S=\sum_{j}^{m} \prod_{k}^{m_{j}} T_{\varphi_{i, k}}$, where $\varphi_{i, k} \in L^{\infty}(D)$, then $S$ is compact if and only if its Berezin transform vanishes at the boundary of the disk. Moreover, they asked if their result could hold if the disk is replaced by other domains in $\mathbf{C}$ which "lack appropriate symmetry." The aim of this paper is to prove, using new techniques, that the same characterization holds when $\Omega$ is a general smoothly bounded multiply-connected planar domain. Such multiply-connected domains "lack appropriate symmetry".

\section{Preliminaries}

Let $\Omega$ be a bounded multiply-connected domain in the complex plane $\mathbf{C}$, whose boundary $\partial \Omega$ consists of finitely many simple closed smooth analytic curves $\gamma_{j}$ $(j=1,2, \ldots, n)$, where $\gamma_{j}$ are positively oriented with respect to $\Omega$ and $\gamma_{j} \cap \gamma_{i}=\varnothing$ if $i \neq j$. We also assume that $\gamma_{1}$ is the boundary of the unbounded component of $\mathbf{C} \backslash \Omega$. Let $\Omega_{1}$ be the bounded component of $\mathbf{C} \backslash \gamma_{1}$, and $\Omega_{j}(j=2, \ldots, n)$ the unbounded component of $\mathbf{C} \backslash \gamma_{j}$, respectively, so that $\Omega=\bigcap_{j=1}^{n} \Omega_{j}$. We use the

Received by the editors May 4, 1999

2000 Mathematics Subject Classification. Primary 47B35.

(C)2000 American Mathematical Society 
symbol $\Delta$ to indicate the punctured disk $\{z \in \mathbf{C}|0<| z \mid<1\}$. Let $\Gamma$ be any one of the domains $\Omega, \Delta, \Omega_{j}(j=2, \ldots, n)$. We call $K^{\Gamma}(z, w)$ the reproducing kernel of $\Gamma$ and we use the symbol $k^{\Gamma}(z, w)$ to indicate the normalized reproducing kernel, i.e. $k^{\Gamma}(z, w)=K^{\Gamma}(z, w) / K^{\Gamma}(w, w)^{\frac{1}{2}}$.

For any $A \in \mathcal{B}\left(H^{2}(\Gamma, d \nu)\right)$ we denote $\tilde{A}$, the Berezin transform of $A$ (see [3] and [2]), where $\tilde{A}(w)=\left\langle A k_{w}^{\Gamma}, k_{w}^{\Gamma}\right\rangle=\int_{\Gamma} A k_{w}^{\Gamma}(z) \overline{k_{w}^{\Gamma}(z)} d \nu(z)$. With these definitions in mind we can state the main theorem:

Theorem 2.1. Let $A$ be an operator in $B\left(H^{2}(\Omega, d \nu)\right)$ which can be written as $A=\sum_{j=1}^{m} \prod_{k=1}^{m_{j}} T_{\varphi_{j, k}}$, where $\varphi_{j, k} \in L^{\infty}(\Omega, d \nu)$. Then $A$ is compact if and only if $\tilde{A}(z) \rightarrow 0$ as $z \rightarrow \partial \Omega$.

We remind the reader (see [6]) that any bounded multiply-connected domain whose boundary consists of finitely many simple closed smooth analytic curves, i.e. a regular domain, is conformally equivalent to a canonical bounded multiplyconnected domain whose boundary consists of finitely many circles. Moreover, it is possible to prove (see [1]) the following

Theorem 2.2. Let $\Omega$ be a regular domain and let $\psi$ be a conformal mapping from $\Omega$ onto $D$. Then $K^{D}(\psi(z), \psi(w)) \psi^{\prime}(z) \overline{\psi^{\prime}(w)}=K^{\Omega}(z, w)$ and the operator $V_{\psi} f=$ $\psi^{\prime} \cdot f \circ \psi$ is an isometry from $L^{2}(D)$ onto $L^{2}(\Omega)$.

If $A \in B\left(H^{2}(\Omega)\right)$ and we define $A_{D} \in B\left(H^{2}(D)\right)$ as $V_{\psi^{-1}} A V_{\psi}$, where $\psi$ is a conformal mapping from $\Omega$ onto $D$, then we can prove the following

Proposition 2.3. $\tilde{A}(z)=\tilde{A}_{D}(\psi(z))$.

Proof. We have, by definition, $\tilde{A}(z)=\left\langle A k_{z}^{\Omega}, k_{z}^{\Omega}\right\rangle=\int_{\Omega} A k_{z}^{\Omega}(w) \overline{k_{z}^{\Omega}(w)} d w$, where $k_{z}^{\Omega}(\cdot)=K_{z}^{\Omega}(\cdot, z) K_{z}^{\Omega}(z, z)^{-\frac{1}{2}}$. Let us take $\psi^{-1}: D \rightarrow \Omega$. Since $\left(J_{\mathbf{R}} \psi^{-1}\right)(\beta)$ is $\left|\left(\psi^{-1}\right)^{\prime}(\beta)\right|^{2}$ and there exists $\zeta \in D$ such that $\psi(z)=\zeta$ we obtain

$$
\tilde{A}(z)=\int_{D}\left(\left(V_{\psi^{-1}} A V_{\psi}\right)\left(V_{\psi^{-1}} k_{\psi^{-1}(\varsigma)}^{\Omega}\right)(\beta) \overline{k_{\psi^{-1}(\varsigma)}^{\Omega}\left(\psi^{-1}(\beta)\right)\left(\psi^{-1}\right)^{\prime}(\beta)} d \beta .\right.
$$

Since Theorem 1.2 implies that

$$
k_{\psi^{-1}(\zeta)}^{\Omega}\left(\psi^{-1}(\beta)\right)=\left|\left(\psi^{-1}\right)^{\prime}(\zeta)\right|\left(\left(\psi^{-1}\right)^{\prime}(\beta) \overline{\left(\psi^{-1}\right)^{\prime}(\zeta)}\right)^{-1} \cdot k_{\zeta}^{D}(\beta),
$$

then

$$
\tilde{A}(z)=\int_{D}\left(A_{D} k_{\zeta}^{D}\right)(\beta) \overline{k_{\zeta}^{D}(\beta)} d \beta ;
$$

it follows that $\tilde{A}(z)=\tilde{A}_{D}(\psi(z))$.

Since every conformal map is an open map, we can conclude that it is enough to prove the theorem when the domain is a canonical bounded multiply-connected domain whose boundary consists of finitely many circles.

\section{The structure of $H^{2}(\Omega)$ and some estimates ABOUT THE BERGMAN KERNEL}

In this section we state well-known facts which we will use in our work; for the proofs the reader can see [1] or [] and [4].

From now on we will assume that $\Omega=\bigcap_{j=1}^{n} \Omega_{j}$, where $\Omega_{1}=\{z \in \mathbf{C}:|z|<1\}$ and $\Omega_{j}=\left\{z \in \mathbf{C}:\left|z-a_{j}\right|>r_{j}\right\}$ for $j=2, \ldots, n$. Here $a_{j} \in \Omega_{1}$ and $0<r_{j}<1$ with $\left|a_{j}-a_{k}\right|>r_{j}+r_{k}$ if $j \neq k$ and $1-\left|a_{j}\right|>r_{j}$. We will indicate with the symbol 
$\Delta$ the punctured disk $\Omega_{1} \backslash\{0\}$. With the symbols $K^{\Omega_{j}}(z, w), K^{\Omega}(z, w), K^{\Delta}(z, w)$ we denote the Bergman kernel on $\Omega_{j}, \Omega$, and $\Delta$ respectively.

Theorem 3.1. There exists an isomorphism $\mathcal{I}: L^{2}(\Delta) \longrightarrow L^{2}\left(\Omega_{1}\right)$ such that $H^{2}\left(\Omega_{1}\right)=\mathcal{I}\left(H^{2}(\Delta)\right)$. Moreover, the Bergman kernels $K^{\Delta}$ and $K^{\Omega_{1}}$ satisfy the equation $K^{\Delta}(z, w)=K^{\Omega_{1}}(z, w)$.

We observe that the previous Theorem and the well-known fact that the reproducing kernel of the unit disk is given by $(1-z \bar{w})^{-2}$ imply that, for $j=2, \ldots, n$, $K^{\Omega_{j}}(z, w)=r_{j}^{2}\left(r_{j}^{2}-\left(z-a_{j}\right) \cdot \overline{\left(w-a_{j}\right)}\right)^{-2}$. Moreover, Theorem 3.1 and Theorem 2.3 and the result of Axler and Zheng imply that if $A=\sum_{j=1}^{m} \prod_{k=1}^{m_{j}} T_{\varphi_{j, k}}$, where $\varphi_{j, k} \in L^{\infty}\left(\Omega_{\ell}\right)$ and $\ell=2, \ldots, n$, then $A$ is compact if and only if its Berezin transform vanishes at the boundary.

We remind the reader that it has been proved that the kernel of $\Omega$ looks like the kernel of $\Omega_{s}$ near $\partial \Omega_{s}$. In fact, if we define $K_{0}^{\Omega}(z, w)=K^{\Omega}(z, w)-\sum_{\ell=1}^{n} K^{\Omega_{\ell}}(z, w)$, where for any $z, w \in \Omega \times \Omega K_{\ell}^{\Omega}(z, w)=K^{\Omega_{\ell}}(z, w)$, we have the following

Lemma 3.2. 1. $K_{0}^{\Omega}$ is conjugate symmetric about $z$ and $w$. For each $w \in \Omega$, $K_{0}^{\Omega}(\cdot, w)$ is conjugate analytic on $\Omega$ and $K_{0}^{\Omega} \in \mathcal{C}^{\infty}(\bar{\Omega} \times \Omega)$.

2. There are neighborhoods $U_{j}$ of $\partial \Omega_{j}(j=1, \ldots, n)$ and a constant $C>0$ such that $U_{j} \cap U_{k}$ is empty if $j \neq k$ and $\left|K^{\Omega}(z, w)-K_{j}^{\Omega}(z, w)\right|<C$ for $z \in \Omega$ and $w \in U_{j}$.

3. $K_{0}^{\Omega} \in L^{\infty}(\Omega \times \Omega)$.

4. There are constants $D>0$ and $M>0$ such that for any $(z, w) \in G_{i} \times \Omega \cup$ $\Omega \times G_{i}$ we have $\left|K^{\Omega}(z, w)\right|<D\left|K_{j}^{\Omega}(z, w)\right|$ and $\left|K_{j}^{\Omega}(z, w)\right|<\left|K^{\Omega}(z, w)\right|+M$.

5. For any $z \in \Omega$ we have $K_{j}^{\Omega}(z, z)<K^{\Omega}(z, z)$.

The next Lemma gives us a good insight into the structure of the space $H^{2}(\Omega)$. This lemma will play a special role in our construction.

Lemma 3.3. For $f \in H^{2}(\Omega)$, we can write it uniquely as $f(z)=\sum_{j=1}^{n}\left(P_{j} f\right)(z)+$ $\left(P_{0} f\right)(z)$ with $P_{j} f \in H^{2}\left(\Omega_{j}\right), P_{0} f \in H^{2}(\Omega) \cap \mathcal{C}^{\infty}(\bar{\Omega}), P_{k}\left(P_{j} f\right)=0$ if $j \neq k$ and there exists a constant $M_{1}$ such that, for $j=0,1, \ldots, n$, we have $\left\|P_{j} f\right\|_{\Omega} \leq\left\|P_{j} f \mid\right\|_{\Omega_{j}} \leq$ $M_{1}\|f\|_{\Omega}$. In particular, if $f \in H^{2}\left(\Omega_{i}\right)$, then $P_{i} f=f$ and $\|f\|_{\Omega_{i}} \leq M_{1}\|f\|_{\Omega}$ for $i=1, \ldots, n$.

Moreover, if $\left\{f_{n}\right\}$ is a bounded sequence in $H^{2}(\Omega)$ and $f_{n} \rightarrow 0$ weakly in $H^{2}(\Omega)$, then $P_{j} f_{n} \rightarrow 0$ weakly on $H^{2}\left(\Omega_{j}\right)$ for $j=1, \ldots, n$ and $P_{0} f_{n} \rightarrow 0$ uniformly on $\Omega$.

\section{Multiply-COnNeCted domains}

The goal of this section is to prove Theorem 2.1. We start with the following

Definition. Let $\Omega=\bigcap_{i=1}^{n} \Omega_{i}$ be a bounded canonical multiply-connected domain. We say that the set of $n$ functions $\mathcal{P}=\left\{p_{1}, \ldots, p_{n}\right\}$ is a $\partial$-partition for $\Omega$ if

1 . For every $j=1, \ldots, n, p_{j}: \Omega \rightarrow[0,1]$ is a Lipschitz, $\mathcal{C}^{\infty}$-function.

2. For every $j=1, \ldots, n$ there exists an $\epsilon_{j}>0$ such that if $U_{\epsilon_{j}}=\left\{\zeta \in \Omega: r_{j}<\right.$ $\left.\left|\zeta-a_{j}\right|<r_{j}+\epsilon_{j}\right\}$, then $p_{j}(\zeta)=1 \forall \zeta \in U_{\epsilon_{j}}$ and $p_{j}(\zeta)=0$ if $\zeta \in U_{\varepsilon_{k}}$ and $k \neq j$.

3. For any $\zeta \in \Omega, \sum_{i=1}^{n} p_{i}(\zeta)=1$.

From now on, in order to make our notation a little simpler, when we use a kernel operator we will denote it by the name of its kernel function. For example, 
the Bergman Projection will be denoted by the symbol $K^{\Omega}$. Finally, we define the operators $Q_{\ell}: L^{2}(\Omega) \longrightarrow L^{2}(\Omega)$, for $\ell=1,2, \ldots, n$, in the following way:

$$
Q_{\ell} f(z)=\int_{\Omega} f(\zeta)\left|K_{\ell}^{\Omega}(\zeta, z)\right| d v(\zeta)
$$

It is important to notice that $\forall \ell=1, \ldots, n, Q_{\ell}$ is a bounded operator (see [7]).

We start with the following

Lemma 4.1. Let $A=\sum_{j=1}^{N}\left(T_{\phi_{j, 1}} \ldots T_{\phi_{j, n_{j}}}\right)$, where $\phi_{j, k} \in L^{\infty}(\Omega)$ for any $j$ and k. If $\mathcal{P}=\left\{p_{1}, \ldots, p_{n}\right\}$ is a $\partial$-partition for $\Omega$ and $R_{s} \in \mathcal{B}\left(H^{2}(\Omega)\right)$, for $s=1, \ldots, n$, is defined as $\sum_{j=1}^{N}\left(K_{s}^{\Omega} M_{\phi_{j, 1} p_{s}} \ldots K_{s}^{\Omega} M_{\phi_{j, n_{j}} p_{s}}\right)$, then the following are equivalent:

1. A is a compact operator;

2. The operator $R_{\ell} P_{\ell}$ is compact for $\ell=1, \ldots, n$.

Proof. If we define $A_{j}$ as $A_{j}=T_{\phi_{j, 1}} \ldots T_{\phi_{j, n_{j}}}$ it follows that $A=\sum_{j}^{N} A_{j}$. Since the Bergman kernel of $\Omega$ is given by $\sum_{s=0}^{n} K_{s}^{\Omega}$ and $\mathcal{P}=\left\{p_{1}, \ldots, p_{n}\right\}$ is a $\partial$-partition for $\Omega$, then we have

$$
A_{j}=\left(\sum_{s=0}^{n} K_{s}^{\Omega}\right)\left(\sum_{t=1}^{n} M_{\phi_{j, 1} p_{t}}\right) \ldots\left(\sum_{s=0}^{n} K_{s}^{\Omega}\right)\left(\sum_{t=1}^{n} M_{\phi_{j, n} p_{t}}\right) .
$$

A simple calculation shows that there exists a compact operator $K_{j}$ such that $A_{j}=K_{j}+\sum_{s=1}^{n} K_{s}^{\Omega} M_{\phi_{j, 1} p_{s}} \ldots K_{s}^{\Omega} M_{\phi_{j, n} p_{s}}$. This implies that

$$
A=\sum_{j=1}^{N} \sum_{s=1}^{n} \prod_{k=1}^{n_{j}} K_{s}^{\Omega} M_{\phi_{j, k} p_{s}}+\sum_{j=1}^{N} \mathcal{K}_{j}
$$

and, if we define $\mathcal{K}=\sum_{j=1}^{N} \mathcal{K}_{j}$ and $R_{s}=\sum_{j=1}^{N} \prod_{k=1}^{n_{j}} K_{s}^{\Omega} M_{\phi_{j, k} p_{s}}$, we have $A=$ $\sum_{s=1}^{n} R_{s}+\mathcal{K}$. Since $\mathcal{K}$ is a sum of compact operators then it is a compact operator. Hence this implies that $A$ is compact if and only if $\sum_{s=1}^{n} R_{s}$ is compact.

We observe that $I=\sum_{\ell=0}^{n} P_{\ell}$ implies

$$
\sum_{t=1}^{n} R_{t}=\sum_{t=1}^{n} R_{t} \sum_{\ell=0}^{n} P_{\ell}=\sum_{t=1}^{n} R_{t} P_{0}+\sum_{t, \ell=1}^{n} R_{t} P_{\ell}
$$

where the $P_{\ell}$ 's are the operators studied in Lemma 3.3.

Claim I. The operator $R_{t} P_{0}$ is compact for $t=1, \ldots, n$.

Proof. Since $R_{t} P_{0}=\sum_{j=1}^{N} K_{t}^{\Omega} M_{\phi_{j, 1} p_{t}} \ldots K_{t}^{\Omega} M_{\phi_{j, n_{j}} p_{t}} P_{0}$, it is enough to prove that for any $n_{j}$ with $j=1, \ldots, N$ the operator $K_{t}^{\Omega} M_{\phi_{j, n_{j}} p_{t}} P_{0}$ is compact. We observe that if $g \in L^{2}(\Omega)$, then

$$
K_{t}^{\Omega} M_{\phi_{j, n_{j}} p_{t}} P_{0} g(z)=\int_{\Omega} K_{t}^{\Omega}(w, z) \phi_{j, n_{j}}(w) p_{t}(w) P_{0} g(w)=Q_{t}\left(\phi_{j, n_{j}} p_{t} P_{0} g\right) .
$$

Let $\left\{f_{n}\right\}$ be a bounded sequence in $H^{2}(\Omega)$ such that $f_{n} \rightarrow 0$ weakly. Lemma 3.3 implies that $P_{0} f_{n} \rightarrow 0$ uniformly on $\Omega$; it follows that $\left\|P_{0} f_{n}\right\|_{\infty} \rightarrow 0$. Therefore $(*)$ and the fact that $Q_{t}$ is bounded imply that

$$
\left\|K_{t}^{\Omega} M_{\phi_{j, n_{j}} p_{t}} P_{0} f_{n}\right\|_{2}=\left\|Q_{t}\left(\phi_{j, n_{j}} p_{t} P_{0} f_{n}\right)\right\|_{2} \leq\left\|P_{0} f_{n}\right\|_{\infty}\left\|Q_{t} \phi_{j, n_{j}} p_{t}\right\|_{2} \rightarrow 0 .
$$

This inequality together with a well-known theorem (see [5, Chap. 5]) implies that the operator is compact. 
Claim II. The operator $R_{s} P_{\ell}$ is compact if $s \neq \ell$ and $s, \ell=1, \ldots, n$.

Proof. Since $R_{s} P_{\ell}=\sum_{j=1}^{N} K_{s}^{\Omega} M_{\phi_{j, 1} p_{s}} \ldots K_{s}^{\Omega} M_{\phi_{j, n_{j}} p_{s}} P_{\ell}$, it is enough to prove that for any $n_{j}$, with $j=1, \ldots, N$, the operator $R_{s, \ell}=K_{s}^{\Omega} M_{\phi_{j, n_{j}} p_{s}} P_{\ell}$ is compact. To do so we consider a bounded sequence $\left\{f_{n}\right\}$ in $L^{2}(\Omega)$ such that $f_{n} \rightarrow 0$ weakly and we prove that $\left\|R_{s, \ell} f_{n}\right\|_{2} \rightarrow 0$. We know that the continuity of $P_{\ell}$ implies that $P_{\ell} f_{k} \rightarrow 0$ weakly on $H^{2}\left(\Omega_{l}\right)$ and $\left\{\left\|P_{\ell} f_{k}\right\|_{\Omega_{\ell}}\right\}$ is bounded by Lemma 3.3. Since it is a sequence of holomorphic functions we know that $\left\{P_{\ell} f_{k}\right\}$ is uniformly bounded on any compact subset of $\Omega_{\ell}$. Therefore the sequence $\left\{P_{\ell} f_{k}\right\}$ is a normal family of functions. Since $P_{\ell} f_{k}(\zeta) \rightarrow 0$ for any $\zeta \in \Omega_{\ell}$, then $P_{\ell} f_{k}$ converges uniformly on any compact subset of $\Omega_{\ell}$ and consequently on $F=\operatorname{supp}\left(p_{s}\right)$. Now we observe that

$$
\left|R_{s, \ell} f_{k}(\zeta)\right| \leq \operatorname{Sup}\left\{\left|P_{\ell} f_{k}(\zeta)\right|: \zeta \in F\right\} \cdot\left|Q_{\ell}\left(\left|\mathcal{X}_{F} \phi_{j, n_{j}} p_{s}\right|\right)(\zeta)\right|
$$

Then, by using the fact that $Q_{\ell}$ is bounded, we have

$$
\left\|R_{s, \ell} f_{k}\right\|_{\Omega} \leq \operatorname{Sup}\left\{\left|P_{\ell} f_{k}(\zeta)\right|: \zeta \in F\right\} \cdot M \cdot\left\|\phi_{j, 1} p_{s}\right\|_{\Omega, 2} \rightarrow 0
$$

and this completes the proof of our claim.

Finally, we observe that Claim I and Claim II imply that if, for any $t, R_{t} P_{t}$ is compact, then $A$ is compact. Moreover, since $P_{t}^{2}=P_{t}, P_{t} P_{s}=0$ if $s \neq t$, and $I$, the identity operator on $H^{2}(\Omega)$, is equal to $\sum_{0}^{n} P_{t}$, then $A$ compact implies that $R_{t} P_{t}$ is compact for any $t$ and this completes the proof of the theorem.

Remark. In the last Theorem we started with the operator

$$
A=\sum_{j=1}^{N}\left(T_{\phi_{j, 1}} \ldots T_{\phi_{j, n_{j}}}\right)
$$

and we have constructed the operator $R_{s}=\sum_{j=1}^{N} K_{s}^{\Omega} M_{\phi_{j, 1} p_{s}} \ldots K_{s}^{\Omega} M_{\phi_{j, n_{j}} p_{s}}$. Since for any $\zeta, z \in \Omega K_{s}^{\Omega}(\zeta, z)=K^{\Omega_{s}}(\zeta, z)$, we know that $R_{s}$ is an element of $B\left(H^{2}\left(\Omega_{s}\right)\right)$. We also know that by construction $\phi_{j, i} p_{s} \in L^{\infty}\left(\Omega_{s}\right)$, so we can construct the following bounded operator

$$
A_{\Omega_{s}}=\sum_{j=1}^{N} K^{\Omega_{s}} M_{\phi_{j, 1} p_{s}} \ldots K^{\Omega_{s}} M_{\phi_{j, n_{j}} p_{s}}
$$

which acts on $H^{2}\left(\Omega_{s}\right)$.

We observe that $R_{s}-A_{\Omega_{s}}$ is compact. In fact, since

$$
A_{\Omega_{s}}=\sum_{j=1}^{N} \prod_{k=1}^{n_{j}} K^{\Omega_{s}} M_{\left(\mathcal{X}_{\Omega}+\mathcal{X}_{\Omega_{s}-\Omega}\right) \phi_{j, k} p_{s}},
$$

it follows that $A_{\Omega_{s}}=R_{s}+\sum_{j=1}^{N} \sum_{k=1}^{2^{n_{j}}-1} T_{j, k}$, where each $T_{j, k}$ is a product of $n_{j}$ Toeplitz operators and at least one of them has the form $K^{\Omega_{s}} M_{\mathcal{X}_{\Omega_{s}-\Omega}} M_{\phi_{j, i} p_{s}}$. Since $\int_{\Omega_{s}} \int_{\Omega_{s}}\left|K^{\Omega_{s}}\right|^{2}\left|\mathcal{X}_{\Omega_{s}-\Omega}\right|^{2}\left|\phi_{j, i} p_{s}\right|^{2} d z d w<\infty, T_{j, k}$ is compact for any $j$ and $k$. Therefore $R_{s}-A_{\Omega_{s}}$ is compact. With the definition of $A_{\Omega_{s}}$ in mind, the fact that $P_{s}\left(H^{2}(\Omega)\right)=H^{2}\left(\Omega_{s}\right)$ and the result proved in the last theorem, we can state the following. 
Corollary 4.2. If $A$ is a member of $B\left(H^{2}(\Omega)\right)$ which can be written as

$$
\sum_{j=1}^{N} \prod_{k=1}^{m_{j}} T_{\phi_{j, k}}
$$

then $A$ is compact if and only if $A_{\Omega_{s}} \in B\left(H^{2}\left(\Omega_{s}\right)\right)$ is compact for $s=1, \ldots, n$.

To prove the main theorem it is important to observe that

Lemma 4.3. Suppose that $\phi \in \mathcal{C}^{\infty}(\bar{\Omega})$ and it is a Lipschitz function; then the operator $\left[P, M_{\phi}\right]$ is compact.

Proof. We observe that $\left[P, M_{\phi}\right]$ is an integral operator, the kernel of which has the form $F(z, w)=(\phi(z)-\phi(w)) K^{\Omega}(z, w)$. Using the same decomposition of $\Omega$ as in Lemma 3.3 we can write that $\Omega=H \cup\left(\bigcup_{\ell=}^{n} G_{\ell}\right)$, where $\forall \ell=1, \ldots, n, G_{\ell}=\left\{z \mid r_{\ell}<\right.$ $\left.\left|z-a_{\ell}\right| \leq r_{\ell}+\epsilon\right\}, \epsilon$ is such that $\forall j \neq k \bar{G}_{k} \cap \bar{G}_{j}=\varnothing$ and $H=\Omega-\left(\bigcup_{j=1}^{n} G_{j}\right)$. Observe that $H \neq \varnothing$. Given such a partition of $\Omega$ we can conclude that $\left[P, M_{\phi}\right]$ is compact if and only if the operator $\sum_{\ell=1}^{n} \mathcal{X}_{G_{\ell}}(z) \mathcal{X}_{G_{\ell}}(w)(\phi(z)-\phi(w)) K^{\Omega}(z, w)$ is a compact operator.

Claim. The operator $S_{\ell}(z, w)=\mathcal{X}_{G_{\ell}}(z) \mathcal{X}_{G_{\ell}}(w)(\phi(z)-\phi(w)) K^{\Omega}(z, w)$ is in the Schatten class $C_{q}$, where $q=p(p-1)^{-1}, p \in(1,2)$ and $\ell=1, \ldots, n$.

Proof. We know (see [9]) that to prove our claim it is enough to show that $\mathcal{S}_{\ell} \in$ $\mathrm{E}^{p}\left(G_{\ell} \times G_{\ell}\right)$ for $1<p<2$. By Lemma 3.2 we know that in $G_{\ell} \times G_{\ell}$ the inequality $\left|K^{\Omega}(z, w)\right|^{p}\left|K^{\Omega_{\ell}}(z, w)\right|^{-p} \leq D^{p}$ holds and since $\phi$ is a Lipschitz function, there exists a constant $M$ such that

$$
\int_{G_{\ell}} \int_{G_{\ell}}|\phi(w)-\phi(z)|^{p}\left|K^{\Omega}(z, w)\right|^{p} d z d w \leq \int_{G_{\ell}} \int_{G_{\ell}} \frac{|z-w|^{p} D^{p} M^{p} r_{\ell}^{2 p} d z d w}{\left|r_{\ell}^{2}-\overline{\left(z-a_{\ell}\right)}\left(w-a_{\ell}\right)\right|^{2 p}} .
$$

If we define $\varsigma=\left(z-a_{\ell}\right) r_{\ell}^{-1}$ and $\omega=\left(w-a_{\ell}\right) r_{\ell}^{-1}$, then we can write

$$
\int_{G_{\ell}} \int_{G_{\ell}} D^{p} M^{p} r_{\ell}^{2 p} \frac{|z-w|^{p} d z d w}{\left|r_{\ell}^{2}-\overline{\left(z-a_{\ell}\right)}\left(w-a_{\ell}\right)\right|^{2 p}}=\int_{\tilde{G}_{\ell}} \int_{\tilde{G}_{\ell}} D^{p} M^{p} r_{\ell}^{p} \frac{|\zeta-\omega|^{p}}{|1-\bar{\zeta} \omega|^{2 p}} d \zeta d \omega,
$$

where $\tilde{G}_{\ell}=\left\{\zeta|1<| \zeta \mid \leq 1+\epsilon r_{\ell}^{-1}\right\}$. Since $\left|(\zeta-\omega)(1-\bar{\zeta} \omega)^{-1}\right|$ is bounded in $\tilde{G}_{\ell} \times \tilde{G}_{\ell}$, we can conclude that $\int_{G_{\ell}} \int_{G_{\ell}}|\phi(w)-\phi(z)|^{p}\left|K^{\Omega}(z, w)\right|^{p} d z d w<\infty$ if and only if

$$
I=\int_{\tilde{G}_{\ell}} \int_{\tilde{G}_{\ell}} \frac{d \zeta d \omega}{|1-\bar{\zeta} \omega|^{p}}<\infty
$$

A simple calculation shows that $I$ is finite if $p<2$; then we can conclude that the operator $S_{\ell}$ is a $C_{q}$ operator. Therefore the operator is a compact operator and this implies that $\left[P, M_{\phi}\right]$ is compact.

Lemma 4.4. Let $\Omega=\bigcap_{t=1}^{n} \Omega_{t}$ be a canonical multiply-connected domain. Suppose that $A \in \mathcal{B}\left(H^{2}(\Omega)\right)$ is an operator which can be written as $A=T_{\phi_{1}} \ldots T_{\phi_{m}}$, where $\phi_{i} \in L^{\infty}(\Omega)$ for $i=1, \ldots, m$ and $p_{s} \in \mathcal{P}$, where $\mathcal{P}=\left\{p_{1}, \cdots, p_{n}\right\}$ is a $\partial$-partition. If we define $A_{p_{s}}$ to be $T_{\phi_{1} p_{s}} \ldots T_{\phi_{m} p_{s}}$, then $\lim _{\zeta \rightarrow \partial \Omega_{s}} \tilde{A}(\zeta)=\lim _{\zeta \rightarrow \partial \Omega_{s}} \tilde{A}_{p_{s}}(\zeta)$.

Proof. We observe that $A=\left(T_{\phi_{1}\left(1-p_{s}\right)}+T_{\phi_{1} p_{s}}\right) \ldots\left(T_{\phi_{m}\left(1-p_{s}\right)}+T_{\phi_{m} p_{s}}\right)$. If we perform all the products we obtain $2^{m}$ terms. One of them is $A_{p_{s}}$. If we write $A_{j}$ for the others $j=1, \ldots,\left(2^{m}-1\right)$ summands, this means that $A=A_{p_{s}}+\sum_{j=1}^{2^{m}-1} A_{j}$. Then it is 
easy to see that each $A_{j}$ can be written as $T_{\psi_{1}^{j}} \ldots T_{\psi_{\ell}^{j}\left(1-p_{s}\right)} \ldots T_{\psi_{m}^{j}}$, where $\psi_{k}^{j} \in L^{\infty}(\Omega)$ for any $j, k$. The point here is that one of the Toeplitz operators which appears in the product has as its symbol a function which vanishes in a neighborhood of $\partial \Omega_{s}$. We claim that for any $j=1, \ldots,\left(2^{m}-1\right)$ there exists a compact operator $K_{j}$ such that $T_{\psi_{1}^{j}} \ldots T_{\psi_{\ell}^{j}\left(1-p_{s}\right)} T_{\psi_{\ell+1}^{j}} \ldots T_{\psi_{m}^{j}}=T_{\psi_{1}^{j}} \ldots T_{\psi_{\ell}^{j}} T_{\psi_{\ell+1}^{j}\left(1-p_{s}\right)} \ldots T_{\psi_{m}^{j}}+K_{j}$. Let us prove this claim. We observe that to prove this claim it is enough to show that the operator $B=T_{\psi_{\ell}^{j}\left(1-p_{s}\right)} T_{\psi_{\ell+1}^{j}}-T_{\psi_{\ell}^{j}} T_{\psi_{\ell+1}^{j}\left(1-p_{s}\right)}$ is compact. Since Lemma 4.3 implies that $B_{1}=T_{\psi_{\ell}^{j}} T_{\left(1-p_{s}\right)}-T_{\psi_{\ell}^{j}\left(1-p_{s}\right)}$ and $B_{1}=T_{\left(1-p_{s}\right)} T_{\psi_{\ell+1}^{j}}-T_{\psi_{\ell}^{j}\left(1-p_{s}\right)}$ are both compact operators, then we can conclude that $B_{1} T_{\psi_{\ell+1}^{j}}$ and $T_{\psi_{\ell}^{j}} B_{2}$ are both compact and this fact proves our claim. If we repeat this process we can conclude that for any $j=1, \ldots,\left(2^{m}-1\right)$ there exists a compact operator $K_{j}$ such that $A_{j}=T_{\psi_{1}^{j} \ldots T_{\psi_{\ell}^{j}} \ldots T_{\psi_{m}^{j}\left(1-p_{s}\right)}}+K_{j}$. If we define $A_{j}^{r}=T_{\psi_{1}^{j} \ldots T_{\psi_{\ell}^{j}} \ldots T_{\psi_{m}^{j}\left(1-p_{s}\right)}}$, then $A-$ $A_{p_{s}}=\sum_{j=1}^{2^{m}-1} A_{j}^{r}+\sum_{j=1}^{2^{m}-1} K_{j}$. Therefore to prove our lemma it is enough to show that $\lim _{\zeta \rightarrow \partial \Omega_{s}}\left\|T_{\psi_{m}^{j}\left(1-p_{s}\right)} k_{\zeta}^{\Omega}\right\|=0$. Since $K_{0}^{\Omega}(w, z)=K^{\Omega}(w, z)-\sum_{\ell=1}^{n} K^{\Omega_{\ell}}(w, z)$ simple calculations imply that

$$
\lim _{z \rightarrow \partial \Omega_{s}} \sum_{\ell \neq s}^{n} \frac{\left\|\psi_{m}^{j}\left(1-p_{s}\right) K_{z}^{\Omega_{\ell}}\right\|_{2}}{\left\|K_{z}^{\Omega}\right\|_{2}}=0 \text { and } \lim _{z \rightarrow \partial \Omega_{s}} \frac{\left\|\psi_{m}^{j}\left(1-p_{s}\right) K_{0, z}^{\Omega}\right\|_{2}}{\left\|K_{z}^{\Omega}\right\|_{2}}=0 .
$$

To complete our proof we need to prove that

$$
\lim _{z \rightarrow \partial \Omega_{s}}\left\|T_{\psi_{m}^{j}\left(1-p_{s}\right)} \frac{K_{z}^{\Omega_{s}}}{\left\|K_{z}^{\Omega}\right\|_{2}}\right\|_{2}=0 .
$$

We have $\left\|T_{\psi_{m}^{j}\left(1-p_{s}\right)} K_{z}^{\Omega_{s}}\right\| K_{z}^{\Omega}\left\|_{2}^{-1}\right\|_{2}^{2} \leq\left\|M_{\psi_{m}^{j}\left(1-p_{s}\right)} K_{z}^{\Omega_{s}}\right\| K_{z}^{\Omega}\left\|_{2}^{-1}\right\|_{2}^{2}$ and this implies that

$$
\left\|T_{\psi_{m}^{j}\left(1-p_{s}\right)} \frac{K_{z}^{\Omega_{s}}}{\left\|K_{z}^{\Omega}\right\|_{2}}\right\|_{2}^{2} \leq\left\|\psi_{m}^{j}\right\|_{\infty}^{2} \frac{\left\|K_{z}^{\Omega_{s}}\right\|_{2}^{2}}{\left\|K_{z}^{\Omega}\right\|_{2}^{2}} \int_{\Omega_{s}}\left|\left(1-p_{s}\right)(w)\right|^{2}\left|k_{z}^{\Omega_{s}}(w)\right|^{2} .
$$

Using the definition of the Berezin Transform we can write that

$$
\left\|\psi_{m}^{j}\right\|_{\infty}^{2} \frac{\left\|K_{z}^{\Omega_{s}}\right\|_{2}^{2}}{\left\|K_{z}^{\Omega}\right\|_{2}^{2}} \int_{\Omega_{s}}\left|\left(1-p_{s}\right)(w)\right|^{2} \frac{\left|K_{z}^{\Omega_{s}}(w)\right|^{2}}{\left\|K_{z}^{\Omega_{s}}\right\|_{2}^{2}}=\left\|\psi_{m}^{j}\right\|_{\infty}^{2} \frac{\left\|K_{z}^{\Omega_{s}}\right\|_{2}^{2}}{\left\|K_{z}^{\Omega}\right\|_{2}^{2}} \tilde{T}_{\left|1-p_{s}\right|^{2}}(z) .
$$

To complete our proof, we observe that $\left\|K_{z}^{\Omega_{s}}\right\|_{2}^{2}\left\|K_{z}^{\Omega}\right\|_{2}^{-2} \leq 1 \forall z \in \Omega$ and $\tilde{T}_{\left|1-p_{s}\right|^{2}}(z)$ $\rightarrow 0$ as $z \rightarrow \partial \Omega_{s}$ since $T_{\left|1-p_{s}\right|^{2}} \in B\left(H^{2}\left(\Omega_{s}\right)\right)$ is compact and this implies that

$$
\lim _{z \rightarrow \partial \Omega_{s}}\left\|T_{\psi_{m}^{j}\left(1-p_{s}\right)} \frac{K_{z}^{\Omega_{s}}}{\left\|K_{z}^{\Omega}\right\|_{2}}\right\|_{2}=0
$$

Remark 1. We observe that $A=\left(\sum_{0}^{n} K^{\Omega_{\ell}}\right) M_{\psi_{1} p_{s}} \ldots\left(\sum_{0}^{n} K^{\Omega_{\ell}}\right) M_{\psi_{m} p_{s}}$. Moreover, since $\forall \ell \neq s$ and $j=1, \ldots, m$ we have that $K_{\ell}^{\Omega} M_{\psi_{j} p_{s}}$ and $M_{\psi_{j} p_{s}} K_{\ell}^{\Omega}$ are compact, therefore $A_{p_{s}}=K_{s}^{\Omega} M_{\psi_{1} p_{s}} \ldots K_{s}^{\Omega} M_{\psi_{m} p_{s}}+\mathbf{k}$, where $\mathbf{k} \in B\left(H^{2}(\Omega)\right)$ is a compact operator.

If we define $A_{s}=K_{s}^{\Omega} M_{\psi_{1} p_{s}} \ldots K_{s}^{\Omega} M_{\psi_{m} p_{s}}$, then we obtain that $\tilde{A}_{p_{s}}(z)=\tilde{A}_{s}(z)+$ $\tilde{\mathbf{k}}(z)$. Therefore $\lim _{z \rightarrow \partial \Omega_{s}} \tilde{A}_{p_{s}}(z)=\lim _{z \rightarrow \partial \Omega_{s}} \tilde{A}_{s}(z)$.

Claim. If $A_{\Omega_{s}} \in B\left(H^{2}\left(\Omega_{s}\right)\right)$, where $A_{\Omega_{s}}=\prod_{\ell=1}^{m}\left(K^{\Omega_{s}} M_{\psi_{\ell} p_{s}}\right)$, and $\tilde{A}_{p_{s}}(z) \rightarrow 0$ as $z \rightarrow \partial \Omega_{s}$, then $\lim _{z \rightarrow \partial \Omega_{s}} \tilde{A}_{\Omega_{s}}(z)=0$.

Before we prove the claim we observe that in the first limit we take the Berezin Transform in $\Omega$ and in the second limit we take the Berezin Transform in $\Omega_{s}$. 
Proof. We know that $\lim _{z \rightarrow \partial \Omega_{s}} \tilde{A}_{p_{s}}(z)=\lim _{z \rightarrow \partial \Omega_{s}} \tilde{A}_{s}(z)$ and that $\tilde{A}_{s}(z)=$ $\left\langle A_{s} k_{z}^{\Omega}, k_{z}^{\Omega}\right\rangle$. A direct calculation implies that $\lim _{z \rightarrow \partial \Omega_{s}}\left\|\left(\sum_{\ell \neq s} K_{\ell, z}^{\Omega}\right)\right\| K_{z}^{\Omega}\left\|_{2}^{-1}\right\|=0$; therefore

$$
\begin{aligned}
\lim _{z \rightarrow \partial \Omega_{s}} A_{s}(z) & =\lim _{z \rightarrow \partial \Omega_{s}}\left\langle A_{s} \frac{K_{s, z}^{\Omega}}{\left\|K_{z}^{\Omega}\right\|_{2}}, \frac{K_{s, z}^{\Omega}}{\left\|K_{z}^{\Omega}\right\|_{2}}\right\rangle \\
& =\lim _{z \rightarrow \partial \Omega_{s}}\left(\frac{\left\|K_{z}^{\Omega_{s}}\right\|_{2}}{\left\|K_{z}^{\Omega}\right\|_{2}}\right)^{2}\left\langle A_{s} k_{z}^{\Omega_{s}}, k_{z}^{\Omega_{s}}\right\rangle .
\end{aligned}
$$

By Lemma 3.2 we know that there exists a constant $D>0$ such that $0<D^{-1} \leq$ $\left\|K_{z}^{\Omega_{s}}\right\|_{2}^{2}\left\|K_{z}^{\Omega}\right\|_{2}^{-2} \leq 1$ if $z$ is close enough to $\partial \Omega_{s}$. Therefore we have proved that $\lim _{z \rightarrow \partial \Omega_{s}} \tilde{A}_{p_{s}}(z)=0$ implies $\lim _{z \rightarrow \partial \Omega_{s}}\left\langle A_{s} k_{z}^{\Omega_{s}}, k_{z}^{\Omega_{s}}\right\rangle=0$. Now we observe that $A_{s}-$ $A_{\Omega_{s}}=K_{s}^{\Omega} M_{\phi_{1} p_{s}} \ldots K_{s}^{\Omega} M_{\phi_{n} p_{s}}-K^{\Omega_{s}} M_{\phi_{1} p_{s}} \ldots K^{\Omega_{s}} M_{\phi_{n} p_{s}}$ is a compact operator. In fact, for any $j$ we have

$$
K_{s}^{\Omega} M_{\phi_{j} p_{s}} f(w)=K^{\Omega_{s}} M_{\phi_{j} p_{s}} f(w)-K_{s}^{\Omega} M_{\mathcal{X}_{\Omega_{s}-\Omega}} M_{\phi_{j} p_{s}} f(w)
$$

and

$$
\int_{\Omega_{s}} \int_{\Omega_{s}}\left|K^{\Omega_{s}}(z, w)\right|^{2}\left|\mathcal{X}_{\Omega_{s}-\Omega}(z)\right|^{2} d z d w<\infty .
$$

It follows that $\lim _{z \rightarrow \partial \Omega_{s}}\left\langle A_{s} k_{z}^{\Omega_{s}}, k_{z}^{\Omega_{s}}\right\rangle=\lim _{z \rightarrow \partial \Omega_{s}}\left\langle A_{\Omega_{s}} k_{z}^{\Omega_{s}}, k_{z}^{\Omega_{s}}\right\rangle$ and this completes the proof of the claim.

Remark 2. If $A$ is an operator on $H^{2}(\Omega)$ which can be written as $\sum_{j=1}^{m} \prod_{k=1}^{m_{j}} T_{u_{j, k}}$, where $u_{j, k} \in L^{\infty}(\Omega), p_{s} \in \mathcal{P}$, where $\mathcal{P}=\left\{p_{1}, \cdots, p_{n}\right\}$ is a $\partial$-partition and $A_{p_{s}}=$ $\sum_{j=1}^{m} \prod_{k=1}^{m_{j}} T_{u_{j, k} p_{s}}$, then $\lim _{\zeta \rightarrow \partial \Omega_{s}} \tilde{A}(\zeta)=\lim _{\zeta \rightarrow \partial \Omega_{s}} \tilde{A}_{p_{s}}(\zeta)$. Moreover, if $A_{\Omega_{s}}$ is defined as before, then it follows that $\lim _{z \rightarrow \partial \Omega_{s}} \tilde{A}_{p_{s}}(z)=0$ implies $\lim _{z \rightarrow \partial \Omega_{s}} \tilde{A}_{\Omega_{s}}(z)=$ 0 .

Proof. Since the operator $A$ can be written as a finite sum of finite products of Toeplitz operators it is enough to rewrite Lemma 4.4 and Remark 1 with the obvious modifications.

Theorem 4.5. Let $\Omega$ be a bounded multiply-connected domain in the complex plain C. If $A$ is an operator on $H^{2}(\Omega)$ which can be written as $\sum_{j=1}^{m} \prod_{k=1}^{m_{j}} T_{u_{j, k}}$, where $u_{j, k} \in L^{\infty}(\Omega)$, then $A$ is a compact operator if and only if $\tilde{A}(\zeta) \rightarrow 0$ as $z \rightarrow \partial \Omega$.

Proof. Since $k_{\lambda}^{\Omega} \rightarrow 0$ weakly in $L^{2}(\Omega)$ and $A$ is compact we have $\left\|A k_{\lambda}^{\Omega}\right\|_{2} \rightarrow 0$ and this implies that $\lim _{\zeta \rightarrow \partial \Omega} \tilde{A}(\zeta)=0$. Now let us suppose that $\tilde{A}(\zeta) \rightarrow 0$; then for any $s=1, \ldots, n$ we have $\lim _{\zeta \rightarrow \partial \Omega_{s}} \tilde{A}(\zeta)=0$. Then Lemma 4.4 implies that $\lim _{\zeta \rightarrow \partial \Omega_{s}} \tilde{A}_{p_{s}}(\zeta)=0$, where $p_{s} \in \mathcal{P}$ and $\mathcal{P}=\left\{p_{1}, \ldots, p_{n}\right\}$ is a $\partial$-partition of $\Omega$.

Using the remarks which follow Lemma 4.4 and the definition of $A_{\Omega_{s}}$ we can conclude that $\lim _{\zeta \rightarrow \partial \Omega_{s}} \tilde{A}_{\Omega_{s}}(\zeta)=0$. Since $A_{\Omega_{s}} \in B\left(H^{2}\left(\Omega_{s}\right)\right)$, the observation after Thorem 3.1 implies that $A_{\Omega_{s}}$ is compact. Since this is true for $s=1, \ldots, n$, Corollary 4.2 implies that $A$ is compact and we are done.

\section{ACKNOWLEDGMENTS}

This paper has been written under the supervision of Professor Lewis A. Coburn. I thank him for his valuable advice and encouragement. 


\section{REFERENCES}

[1] J. Arazy, Membership of Hankel Operators on Planar Domains in Unitary Ideals, Analysis at Urbana, vol.1, London Math. Soc. Lecture Notes Ser. 137, Cambridge University Press, 1989, 1-40. MR 90g:47048

[2] S. Axler and D. Zheng, Compact Operators via the Berezin Transform, Indiana Univ. Math. J. 47 (1998), 387-400. MR 99i:47045

[3] F. A. Berezin, Covariant and Contravariant Symbols of Operators, Math. USSR Izv. 36 (1972), 1117-1151. MR 50:2996

[4] V. Bergman, The Kernel Function and the Conformal Mapping, AMS Math. Surveys 5, 1950.

[5] R. Douglas, Banach Algebra Techniques in Operator Theory, Academic Press, 1972. MR 50:14335

[6] G. M. Goluzin, Geometric Theory of Functions of a Complex Variable, Trans. of Math. Monographs 26 Providence, R.I., 1969. MR 40:308

[7] L. Huiping, Hankel Operators on the Bergman Space of Multiply-Connected Domains, J. Oper. Theory 28 (1992), 321-335. MR 95d:47029

[8] N. Kerzman, The Bergman Kernel Function, Differentiability at the Boundary, Math. Ann. 195 (1972), 149-158. MR 45:3762

[9] B. Russo, On the Hausdorff-Young Theorem for Integral Operators, Pacific J. of Math. 68 (1977), 241-252. MR 58:17974

Department of Economics, University of California at Berkeley, Evans Hall, BerkeLey, California 94720

E-mail address: raimondo@econ.berkeley.edu 